\title{
Modulation of BACE1 activity by chemically modified aptamers
}

\author{
Cécile Gasse, ${ }^{*[a]}$ Marwa Zaarour, ${ }^{[a]}$ Sam Noppen, ${ }^{[b]}$ Mikhail Abramov, ${ }^{[c]}$ Philippe Marlière, ${ }^{[d]}$ Sandra \\ Liekens, ${ }^{[b]}$ Bart De Strooper, ${ }^{[e]}$ Piet Herdewijn*[a, c]
}

\begin{abstract}
A modified DNA aptamer was developed that binds BACE1, a therapeutic target involved in Alzheimer's disease. This ssXNA not only tightly binds to BACE1 but also inhibits its protease activity in vitro in the same range as a previously described unmodified aptamer. We report the in vitro selection of functional oligonucleotides using two nucleobase modifications: 5 -chlorouracil and 7-deazaadenine. The nucleoside analog 5-chloro-2'deoxyuridine has already been explored to replace thymidine in a chemically modified genome of a bacterium. Thus, 5-chlorouracil modification is a good candidate to support genetic transfer in vivo as well as functional activity.
\end{abstract}

\section{Introduction}

Halogenated uracil residues enjoyed a revival in interest over the last few years from a genetical and biochemical point of view. 5-chlorouracil (5-CIU) was previously synthesized in the 1950's but has not been extensively studied as much as other halogenated pyrimidine derivatives, possibly because potential therapeutic applications were not visible. ${ }^{[1]}$

More recently, Marlière et al. brought the chlorouracil modification to the forefront of synthetic biology by challenging cells to replicate with this artificial nucleobase instead of the natural thymine ${ }^{[2]}$ For that purpose, they engineered an $E$. coli strain that required exogenous thymine for growth. $98.5 \%$ of its

[a] Dr. C. Gasse, M. Zaarour, Prof. P. Herdewijn

Univ Evry /CNRS-UMR8030 - Laboratoire iSSB /CEA, DRF, IG, Genoscope / Université Paris-Saclay

5 rue Henri Desbruères, Évry 91030, France

E-mail: cecile.gasse@univ-evry.fr; piet.herdewijn@kuleuven.be

[b] S. Noppen, Prof. S. Liekens

Department of Microbiology and Immunology, Rega Institute for

Medical Research, Laboratory of Virology and Chemotherapy,

KU Leuven, University of Leuven

Herestraat 49, postbus 1043, B-3000 Leuven, Belgium

[c] Dr. M. Abramov, Prof. P. Herdewijn

Medicinal Chemistry, Rega Institute for Medical Research,

KU Leuven, University of Leuven

Herestraat 49, B-3000 Leuven, Belgium

[d] Dr. P. Marlière

The European Syndicate of Synthetic Scientists and Industrialists (TESSI)

81 rue Réaumur 75002 Paris, France

[e] Prof. B. De Strooper

KU Leuven-VIB Center for Brain \& Disease Research, VIB, 3000

Leuven, Belgium

Department of Neurosciences, Leuven Institute for Neuroscience and Disease, KU Leuven, 3000 Leuven, Belgium

Dementia Research Institute UK, University College London, Queen Square, WC1N 3BG London, UK

Supporting information for this article is given via a link at the end of the document. genome contained 5-chlorodeoxyuridine in place of genomic deoxythymidines. This seminal work was the first example in the construction of biological systems composed of xenonucleic acids (XNAs) through evolutionary pressure. The choice of 5$\mathrm{CIU}$ was in part based on the fact that $5-\mathrm{ClU}$ or 5 -CldU were well known for incorporation into $\mathrm{DNA}^{[3-7]}$ and could form a biophysically stable base pair with adenine. Further investigations into the development of artificial genomes were carried out by using fully nucleobase-modified DNA with 5chloro-2'-deoxyuridine, 7-deaza-2'-deoxyadenosine, 5-fluoro-2'deoxycytidine, and 7-deaza-2'deoxyguanosine ${ }^{[8]}$ Originally, the 7-deaza-2'-deoxyadenosine (or tubercidin) was characterized for antiviral, antitrypanosomal, and antifungal functions. ${ }^{[9,10]}$ The choice of 5-chlorouracil and its base-pairing partner, 7-deaza-2'deoxyadenosine as a new base-pair couple relied on their chemical stability and their physicochemical properties. ${ }^{[11,12]}$ The resulting XNAs were shown to be replicated in vitro by the Taq polymerase under polymerase chain reaction (PCR) conditions on very long templates ( $>2000$ nucleotides) and to serve in vivo as artificial genetic templates along with a 5-fluorocytosine: 7deazaguanine base pair. These findings went along with previous studies which demonstrated that alternative backbone modifications, such as triazole units, replacing the natural phosphodiester bond could also assume this function. ${ }^{[13,14]}$ Moreover, the implementation of a modified information system able to sustainably replicate was firstly conducted by using a single base pair system in E. coli. ${ }^{[15]}$

In the present work, we sought to explore the potential of such a redesigned base-pair system in the applied context of functional nucleic acids. In a long-term goal, production of modified nucleic acids with a dedicated function could be accomplished by using living systems tolerant to chemical modifications, as proven by the engineered $E$. coli growing on 5-CIU. Aptamers are singlestranded synthetic oligonucleotides that can adopt well-defined three dimensional structures and thus bind specifically and selectively to a target molecule. ${ }^{[16]}$ They can be generated by applying the SELEX procedure (Systematic Evolution of Ligand by Exponential enrichment) from libraries of randomized singlestranded DNA or RNA molecules with a predetermined diversity. Recent developments have been oriented to increase the chemical diversity in the scaffold of aptamers which in turn will enhance their stability towards nuclease degradation and improve their binding affinity, especially towards hydrophobic or positively charged targets by using chemically-modified nucleotides during the selection protocol. ${ }^{17-24]}$ The same approach has been applied for catalytic nucleic acids. ${ }^{[25-28]}$ We decided to use this chemistry to tackle the aspartyl protease beta-secretase 1 (BACE1), an important drug target in Alzheimer's Disease (AD). ${ }^{[29]}$ Cerebral accumulation of amyloid $\beta$ peptide $(A \beta)$ is thought to be central at an early stage in the pathogenesis of $A D$. This peptide is generated by a sequential cleavage of the amyloid precursor protein (APP) by $\beta$ - and $\gamma^{-}$

For internal use, please do not delete. Submitted_Manuscript 
secretases. BACE1 has been identified as the $\beta$-secretase involved in APP processing and is thus considered as an attractive drug target for potential $A D$ treatment. The feasibility of the approach has been validated by the selection of RNA aptamers recruited to the cytoplasmic domain of BACE ${ }^{[30]}$ and more recently DNA aptamers against the ectodomain of BACE1 containing its catalytic site. ${ }^{[31]}$ In our current work, we describe the in vitro selection of aptamers against BACE1 using an alternative base pair system where the thymidine was substituted by a 5 -CldU and adenosine by its 7-deaza analogue (Figure 1). ${ }^{[32,33]}$ We clearly demonstrate that the incorporation of these nucleobase modifications into the random oligonucleotide library, significantly modified the outcome of the selection process against BACE1. Binding and modulatory activity of the newly chemically modified aptamers are also discussed. This selection experiment represents a step towards the crafting of modified genetic systems capable of modulating enzymes involved in $A D$ and could be expanded to other neurodegenerative diseases.
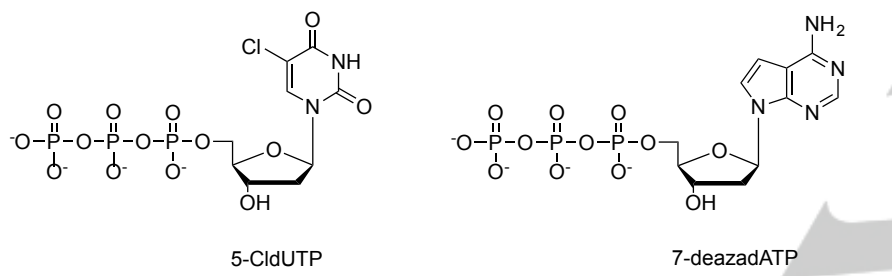

Figure 1. Chemical structure of the modified nucleotides used during SELEX processes.

\section{Results and Discussion}

\section{Amplification with modified triphosphate precursors}

We chose the D1 DNA library comprising a 49 nucleotide-long random region as a template for amplification since this library has displayed good results in previous SELEX processes. ${ }^{[34-36]}$ Similar yields of PCR amplification could be observed by agarose gel analysis when either the modified 5-CldUTP or the corresponding natural dTTP were used in PCR with the Taq polymerase. This further confirmed the potential of 5-CldUTP to serve as precursor for the construction of modified oligonucleotide libraries in lieu of dTTP. On the other hand, while the combination of 5-CldUTP and 7-deazadATP still produced significant PCR amplification, the yields were rather depleted compared to when the natural dNTPs were used (Supplementary data, S1).

\section{In vitro selection}

We applied the procedure described by Mayer et al. for the generation of sSDNA BACE1 aptamers using streptavidin-biotin chemistry to separate bound from unbound DNA species during the selection process. ${ }^{[3]}$ In iterative cycles of selection, the library of SSDNA molecules with a random region flanked by defined primer binding sites was incubated with the biotinylated target protein (BACE1) immobilized on streptavidin-coated magnetic beads. (Scheme 1) Non-binding sequences were removed by washing steps while the bound species were eluted and amplified by PCR. The PCR products were captured on streptavidin magnetic beads via the biotinylated "antisense" strand. After washes of the beads, the unbiotinylated "sense" strands (i.e. the strands used for selection) were eluted from the beads by $\mathrm{NaOH}$ addition. This generated an enriched population of modified ssDNAs which represented the starting point for the next selection cycle.

We carried out two selection experiments, one using 5-CldUTP as sole modification (SELEX A), while in the second approach both natural dTTP and dATP were replaced by 5-CldUTP and 7deazadATP, respectively (SELEX B).

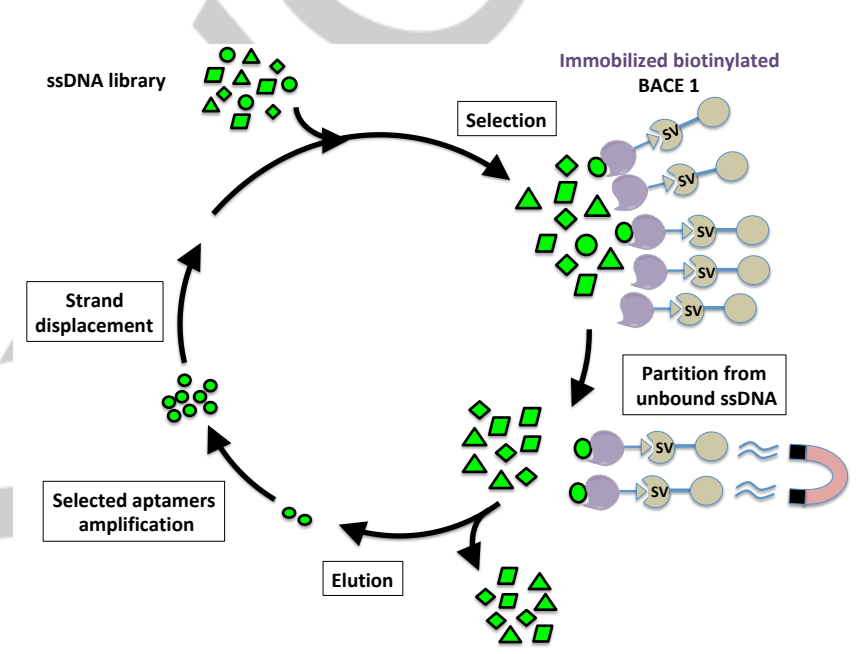

Scheme 1. SELEX strategy to identify anti-BACE 1 aptamers. A random ssDNA library is incubated with the intended target and separation of nonbinding sequences from bound sequences is achieved by using streptavidin (SV)-coated magnetic beads. The bound sequences are then eluted and amplified. Alkaline denaturation of the dsDNA leads to a new ssDNA enriched population ready for the next selection round.

Prior to selection, BACE1 was biotinylated and coupled to streptavidin-coated magnetic particles which were then used directly in the in vitro selection experiment. We determined by Western Blot that a 5-fold Sulfo-NHS-LC-Biotin molar excess of biotinylation reagent was the best experimental condition to obtain high proportions of biotinylated vs. non-biotinylated protein $(\sim 1 / 3)$. Ideally, this would prevent a biotin 'overload' of BACE1 which could mask epitopes of interest during the selection with modified ssDNA. (Supplementary data, S2) The ratio of the total single stranded library to the target was evaluated to be approximatively $26: 1$. The quality of the PCR products was evaluated by agarose gel electrophoresis analysis $(3 \%)$, and was considered successful when a properly sized XNA band was observed by UV monitoring. In this context, we observed that the quality of the XNA band improved during SELEX. Twelve iterations of SELEX were performed for both SELEX $A$ and $B$. The stringency of the selection process was gradually increased (see Table 1). Variables affecting the 
stringency included increasing the amount of wash steps and decreasing the amount of BACE 1 present on the beads.

Table 1. Stringency conditions applied during the SELEX process.

\begin{tabular}{|c|c|c|c|c|c|}
\hline \multicolumn{3}{|c|}{$\begin{array}{l}\text { SELEX with 5-CldUTP instead of } \\
\text { dTTP (SELEX A) }\end{array}$} & \multicolumn{3}{|c|}{$\begin{array}{l}\text { SELEX with 5-CldUTP instead of } \\
\text { dTTP and } 7 \text {-deazadATP instead of } \\
\text { dATP (SELEX B) }\end{array}$} \\
\hline Round & $\begin{array}{l}\text { Washing } \\
\text { cycles }\end{array}$ & $\begin{array}{l}\text { BACE1- } \\
\text { coated } \\
\text { beads }\end{array}$ & Round & $\begin{array}{l}\text { Washing } \\
\text { cycles }\end{array}$ & $\begin{array}{l}\text { BACE1- } \\
\text { coated } \\
\text { beads }\end{array}$ \\
\hline 1 & 1 & $0.4 \mathrm{mg}$ & 1 & 1 & $0.4 \mathrm{mg}$ \\
\hline 2 & 2 & $0.4 \mathrm{mg}$ & 2 & 1 & $0.4 \mathrm{mg}$ \\
\hline 3 & 2 & $0.4 \mathrm{mg}$ & 3 & 2 & $0.4 \mathrm{mg}$ \\
\hline 4 & 4 & $0.33 \mathrm{mg}$ & 4 & 2 & $0.4 \mathrm{mg}$ \\
\hline 5 & 4 & $0.33 \mathrm{mg}$ & 5 & 3 & $0.4 \mathrm{mg}$ \\
\hline 6 & $\begin{array}{l}4-5 \mathrm{~min} \\
4-5 \mathrm{~min}\end{array}$ & $0.33 \mathrm{mg}$ & 6 & 3 & $0.4 \mathrm{mg}$ \\
\hline 7 & $\begin{array}{l}\text { 4-5min; } \\
4-5 \min \end{array}$ & $0.25 \mathrm{mg}$ & 7 & 4 & $0.4 \mathrm{mg}$ \\
\hline 8 & $\begin{array}{l}4-5 \min \\
4-5 \min \end{array}$ & $0.25 \mathrm{mg}$ & 8 & 4 & $0.4 \mathrm{mg}$ \\
\hline 9 & $\begin{array}{l}4-5 \min \\
4-5 \min \end{array}$ & $0.2 \mathrm{mg}$ & 9 & 5 & $0.4 \mathrm{mg}$ \\
\hline 10 & $\begin{array}{l}4-5 \min \\
4-5 \min \end{array}$ & $0.2 \mathrm{mg}$ & 10 & 5 & $0.4 \mathrm{mg}$ \\
\hline 11 & $\begin{array}{l}4-5 \mathrm{~min} \\
4-5 \mathrm{~min}\end{array}$ & $0.13 \mathrm{mg}$ & 11 & 6 & $0.06 \mathrm{mg}$ \\
\hline 12 & $\begin{array}{l}4-5 \mathrm{~min} \\
4-5 \mathrm{~min}\end{array}$ & $0.13 \mathrm{mg}$ & 12 & 6 & $0.06 \mathrm{mg}$ \\
\hline
\end{tabular}

It is noteworthy to mention that all the selection experiments were carried out at the non-optimal $\mathrm{pH}$ of 7.4 and several attempts to perform SELEX at a pH of 4.5 (the optimum for BACE1 activity) failed due to absence of immobilization of the target on the magnetic beads. Indeed, estimation of BACE1 biotinylation by Western blot and immobilization on streptavidincoated magnetic particles clearly revealed that BACE1 was not detected on the beads at such a low $\mathrm{pH}$. The Streptavidin MagneSphere Paramagnetic Particles properties did not appear to be affected at this low $\mathrm{pH}$ since the yields of SsDNA purification from biotinylated PCR were comparable to those obtained at $\mathrm{pH}$ 7.4. In addition, the linker between BACE1 and the biotin is composed of stable amide bonds and the antibody used to detect BACE1 was able to identify the intended protein in the supernatant fraction at this $\mathrm{pH}$. We rationalized that failure to carry out a SELEX with this protocol at a pH of 4.5 might be due to a lack of accessibility of the biotin to the streptavidin linked. This results from a subtle conformation modification of BACE1 at this acidic $\mathrm{pH}$, since the $\mathrm{pH}$-dependent flap dynamics that controls substrate accessibility did not seem to be drastic. ${ }^{[39]}$ When the immobilization of the target was performed at $\mathrm{pH} 7.4$ but the $\mathrm{pH}$ was maintained at 4.5 during the SELEX process, no amplification was detected after a first round of selection. Another SELEX strategy without BACE1 biotinylation was then performed where the complexes of bound ssXNA to the target protein were separated from unbound reagents by filtration. ${ }^{[39]}$ Unfortunately, no amplicon was obtained after the 11 th round of selection despite several attempts.

\section{Cloning and sequencing analysis}

After 12 rounds of selection, PCR products were directly cloned and approximately one hundred positive clonal colonies were sequenced for both SELEX $A$ and $B$ to examine the diversity of molecules that remained in the pool. PCR amplicons from round 7 (SELEX A) and 10 (SELEX B) were equally treated because of an unexpected loss of ssXNA during alkaline denaturation prior to the 8th round of the selection process. Examination of the sequences of the clones from SELEX A (with 5-CIU as modified nucleobase) revealed a major family of selected ssXNA represented by 8 sequences differing only by one to three nucleotides in the 49 nucleotide long randomized region. In addition, two other sequences were represented twice while all the remaining clones were unique sequences (Supplementary data, S3.1). ${ }^{[40]}$ Interestingly, when we analysed the percentage of nucleotide frequency over all the sequences identified from SELEX A, we observed that $5-\mathrm{CIU}$ was significantly the most represented nucleobase with $38.5 \%$ compared to only $14.8 \%$ for A, $15.2 \%$ for $C$ and $31.5 \%$ for $G$. All the identified ssXNA sequences were synthesized by large-scale PCR and directly screened for activity on BACE1 through a MBP-C125APPsw assay. Surprisingly, of all the sequences only one stemming from the major family was active with a $30 \%$ inhibitory effect at $0.25 \mu \mathrm{M}$. Twenty other clones were found to have an inhibitory effect comprised between 17 and $45 \%$ at higher oligonucleotide concentrations ranging from 0.26 to $0.86 \mu \mathrm{M}$. Another sequence was a strong activator and all the remaining sequences had no effect on BACE1. Unexpectedly, no particular common motif raised from the 21 ssXNA active clones through a Muscle multiple sequence alignment (Supplementary data, S3.2). Overall, all the identified sequences were different except for clones 12.6/23 (seq $n^{\circ} 39$ ) and 12.7/13 (seq $n^{\circ}$ 93) which were $93.9 \%$ identical. Examination of the cloned sequences stemming from SELEX B (with both 5-CIU and 7-deazaA as modified nucleobases) did not lead to the identification of clear families (Supplementary data, S3.3). However, almost half of the sequences (44 of 98) were primer dimer sequences (GCCTGTTGTGAGCCTCCTAACATGCTTATTCTTGTCTCCC) that most likely originate from a PCR amplification artefact rather than a true evolution of the sequence population during SELEX. Among the 56 remaining sequences, six were identical in pairs. The percentage of the nucleotides frequency over all these sequences was different from that of SELEX $A$ since $G$ was significantly the most represented nucleobase with nearly $36 \%$ compared to $26 \%$ for 5 -CIU, and $19 \%$ for both 7 -deazaA and C. Multiple local alignment of the sequence obtained from SELEX $A$ and $B$ did not reveal any identical sequence but three common

For internal use, please do not delete. Submitted_Manuscript 
motifs (Supplementary data, S4.2). Following the sequence analysis, a few clones from SELEX B were synthesized by PCR and directly tested for activity using a FRET-based assay. The choice of the few selected clones was mainly based on the finding of the aforementioned common motif by the MEME program analysis tool (Supplementary data, S4). ${ }^{[41]}$

The SELEX performed at $\mathrm{pH} 4.5$ with $5-\mathrm{CIU}$ as a modified nucleobase was also analyzed and PCR amplicons stemming from rounds 7 and 10 were cloned, sequenced and tested for activity. In this selection experiment, neither a clear sequence family nor a common motif could be identified within the sequences of the different clones which was expected due to the lack of sequence convergence. The percentage of the nucleotide frequency over all the sequences revealed that $G$ was again significantly the most represented nucleobase with a value of $34.4 \%$ compared to $26.5 \%$ for 5 -CIU, $20.5 \%$ and $18.6 \%$ respectively for $A$ and $C$. Multiple local alignment of the sequences from SELEX A (CIU), SELEX B (5-CIU and 7deazaA) and SELEX at $\mathrm{pH} 4.5(5-\mathrm{CIU})$ did not lead to the identification of any entire common sequence or any relevant common motif between the three SELEX processes.

\section{Activity and affinity studies}

\section{ssXNA production}

ssXNA were produced in vitro by PCR using the intended recombinant plasmids as templates and carrying out strand displacement by alkaline denaturation and with the use of magnetic streptavidin beads as previously described. This method was validated by comparison with a PAGE purification of asymmetric PCR product. In the asymmetric PCR method, one of the primers (sl-REV-D1), consists of a repeating 5'-GC rich reverse sequence with the ability to form stem-loop structures and contains the 3'-complement sequence of the template. Therefore, the resulting PCR products exhibited two bands with different electrophoretic mobilities in denatured gel electrophoresis; the band with the highest mobility corresponds to the single stranded DNA template while the slower moving band corresponds to the extension product of sl-REV-D1 with a reverse repeating sequence of 30 nucleotides longer than the template. ${ }^{[42]}$ ssXNA resulting from the method using magnetic separation or from the asymmetric PCR method were cloned and sequenced. Following purification by magnetic separation of the ssXNAs we observed one mutation in every 25 analyzed clones. The mutation rate was higher when the asymmetric PCR method was used (one mutation every 22 analyzed clones). The magnetic separation method was then selected for ssXNA production considering the faster protocol and the higher yield of ssXNA recovered for a similar quality of the sequences. Despite the fact that 5-CldUTP residues were described to pair with dATP but also with dGTP, [7] we didn't observe significant misincorporation in place of 5-CIUTP residues (Supplementary data S7). This is consistent with a recent work where the basepairing abilities of oligonucleotides bearing one or three modified nucleosides were investigated showing the proper incorporation of modified dNTPs. ${ }^{[43]}$

\section{MBP-C125APPsw assay}

The effect of ssXNA on BACE1 was determined by application of an assay developed by Sinha et al. ${ }^{[44]}$ Briefly, the exogenous recombinant maltose-binding protein MBP fused to APPsw (571695aa) was used as a substrate and its cleavage product released by the effect of BACE1 was detected by ELISA. This assay was used as a first screen to test the activity of the different ssXNA sequences resulting from SELEX A. From the one hundred and thirteen sequences that were tested, twenty displayed a moderate inhibition ranging from $17 \%$ to $45 \%$ in the tested concentration range $(0.26-0.86 \mu \mathrm{M})$. These preliminary results were the starting point for more in-depth affinity studies and activity characterization.

\section{Binding assay}

We next investigated the binding of the twenty active ssXNAs to BACE1 by surface plasmon resonance studies (SPR). Among the twenty XNA sequences tested at $100 \mathrm{nM}$ with the highest density of BACE1 (3136 RU), only ssXNA ${ }^{\circ} 71$ and the starting library were interacting significantly with BACE1. When BACE1 was directly immobilized on the chip, a $K_{D}$ calculation was not possible because of very slow dissociation most likely due to avidity or more complex binding interaction than the 1:1 binding model. We then decided to perform kinetic studies with immobilized ssXNA in order to avoid avidity limitation. Biotinylated ssXNAs were immobilized on a streptavidin SA sensor chip with BACE1 as analyte. One representative SPR experiment with the responses for SsXNA $n^{\circ} 71$ over time, its unmodified counterpart 71T (with $\mathrm{T}$ nucleobase instead of 5$\mathrm{CIU}$ ) and the initial library is shown in Figure 2 for BACE1 concentrations from 0.156 to $10 \mu \mathrm{g} / \mathrm{ml}$.
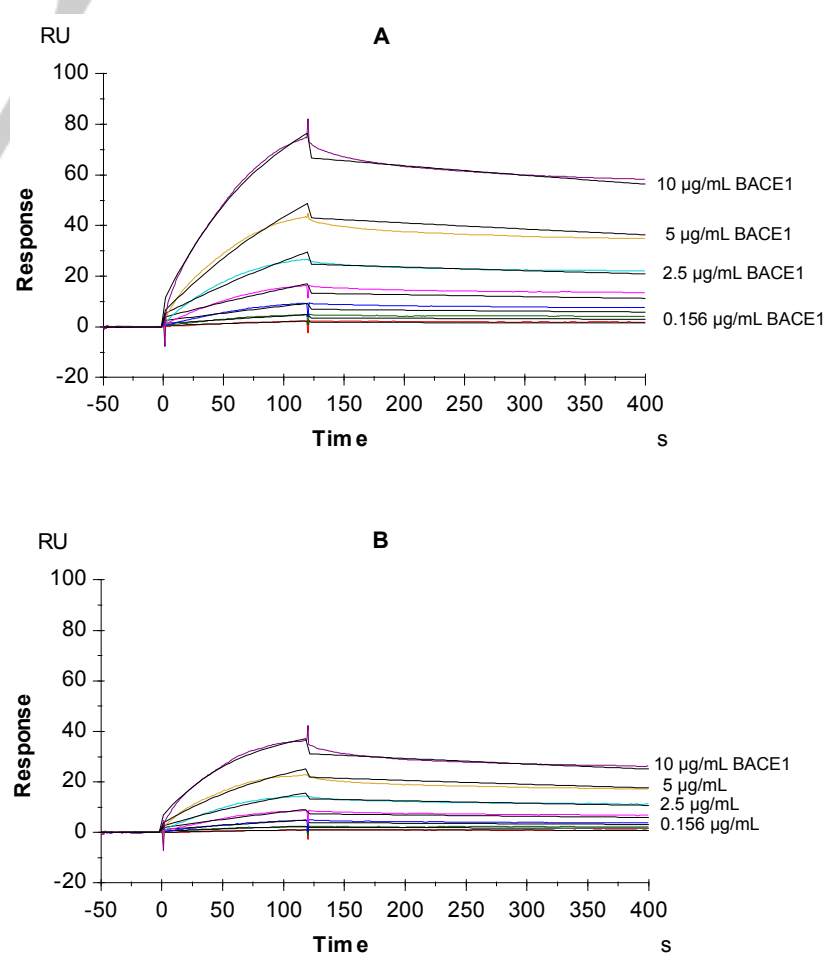

For internal use, please do not delete. Submitted_Manuscript 


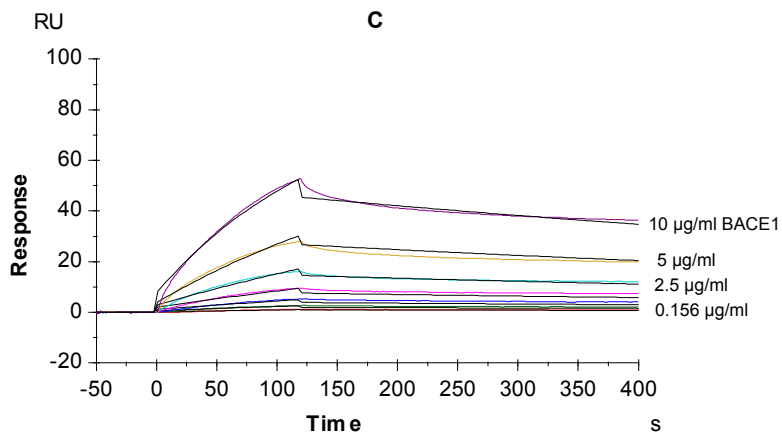

Figure 2. Sensorgrams were obtained from BACE1 injection over the immobilized biotinylated sequences $A: 71, B: 71 T$ and $C$ : Library. The $K_{D}$ values for $71,71 \mathrm{~T}$ and the starting library are $12.0 \pm 1.2 \mathrm{nM}, 10.3 \pm 3.7 \mathrm{nM}$ and $31.0 \pm 5.1 \mathrm{nM}$, respectively.

An apparent equilibrium dissociation constant $\mathrm{K}_{\mathrm{D}}$ and kinetic rate constants ( $\mathrm{K}_{\mathrm{n}}$ and $\mathrm{K}_{\text {off }}$ ) were calculated by fitting the experimental data using the 1:1 binding model (Supplementary data, S5). Strikingly, all three sequences displayed remarkably high affinity for BACE1 $\left(12.0 \pm 1.2 \mathrm{nM}\right.$ for clone $\mathrm{n}^{\circ} 71,10.3 \pm 3.7$ $\mathrm{nM}$ for clone $\mathrm{n}^{\circ} 71 \mathrm{~T}$ and $31.0 \pm 5.1 \mathrm{nM}$ for the starting library). A slightly higher association rate constant (kon) and lower dissociation rate constant $\left(k_{\text {off }}\right)$ resulted in a 2.5 -fold higher affinity of 71 and $71 \mathrm{~T}$ compared to the library (Supplementary data, S5). The high affinity of the library for BACE1 could reflect a sequence bias of the starting pool with an overrepresentation of strong binders which are most likely non-specific. Lastly, all binding interactions were lost at physiological $\mathrm{pH}$. Many SELEX endeavors making use of streptavidin magnetic beads in the selection protocol and especially cell-SELEX have to face considerable non-specific binding to these targets. ${ }^{[45]}$ The addition of a counter selection against the matrix used for target immobilization should in theory reduce non-specific binding to the matrix. Fortunately, no binding to streptavidin nor spermidine was observed in our case (data not shown).

\section{Fluorescence resonance energy transfer (FRET) assay}

Dose-response studies were then conducted with an assay that did not require any specific antibodies and that could be adapted for small volumes. The assay is based on a method of fluorescence resonance energy transfer (FRET), in which the fluorescence signal is observed after a smaller peptidic substrate is cleaved by BACE1. First, a decrease in BACE1 activity of $70,4 \pm 5,2 \%$ ( $n=3$ independent experiments) was observed when the assay buffer was supplemented with $\mathrm{NaCl}$, $\mathrm{KCl}$ and $\mathrm{MgCl}_{2}$. This is possibly due to an alteration of the secondary structure of the protein. ssXNA 71, a randomly generated sequence variant of 71 , and $71 \mathrm{~T}$, were tested in this assay and compared to the previously identified DNA aptamer Apt_A1 against BACE1. ${ }^{[31]}$ In addition, sequences 39 and 93, extracted from the major class of sequences isolated after 12 rounds in SELEX A, the chemically synthesized analogue of 93 depleted of the primer regions and the sequence 118 selected during SELEX B with two modified nucleobases were also assessed. (Table 2)

Table 2. Sequences of selected ssXNA. $X=5-\mathrm{CIU}$ and $P=7$-deazaadenine motif 1 is highlighted in red, motif 2 in cyan, motif 3 in green.

\begin{tabular}{|c|c|}
\hline & Sequences \\
\hline 71 & $\begin{array}{l}\text { gcctgttgtgagcctcctaacGGCGXAGGGXXXGXXGXCXCXAXXXCXXXG } \\
\text { XAXXGXGXXXXXXCGCXatgcttattcttgtctccc }\end{array}$ \\
\hline $71 \mathrm{~T}$ & $\begin{array}{l}\text { gcctgttgtgagcctcctaacGGCGTAGGGTTTGTTGTCTCTATTTCTTTG } \\
\text { TATTGTGTTGTGTTCGCTcatgcttattcttgtctccC }\end{array}$ \\
\hline $\begin{array}{l}\text { Ran } \\
\text { dom } \\
71\end{array}$ & $\begin{array}{l}\text { gcctgttgtgagcctcctaacXCXXGXGCXCGXXGXCCAXGXGGXXGXGGA } \\
\text { XXXGXXGCXXAXXXXXcatgcttattcttgtctccC }\end{array}$ \\
\hline 39 & $\begin{array}{l}\text { gcctgttgtgagcctcctaacGCACCGGXCGCAGGXXXAGCAAGGXCACAA } \\
\text { GCXGGCAXCAAGGXXGCXXcatgcttattcttgtctcCC }\end{array}$ \\
\hline 93 & $\begin{array}{l}\text { gcctgttgtgagcctcctaacGCACCGGXCGCAGGXXXAGCAAGGXXACAA } \\
\text { GCXGGCAXXAAGGXXGCXCcatgcttattcttgtctcCC }\end{array}$ \\
\hline $\begin{array}{l}93 \\
\mathrm{TL}\end{array}$ & GCACCGGXCGCAGGXXXAGCAAGGXXACAAGCXGGCAXXAAGGXXGCXC \\
\hline 118 & $\begin{array}{l}\text { gcctgttgtgagcctcctaacGCPCXCXGGXCGCPXGXGGPGXPXCCGPXX } \\
\text { XGGXXGPGXXCGXXXGCXCcatgcttattcttgtctcCC }\end{array}$ \\
\hline $\begin{array}{l}\text { Apt_- } \\
\text { A1 }\end{array}$ & $\begin{array}{l}\text { gcaatggtacggtacttCCGTCATCAGCTTGTGATGTGGATGCGAACTGca } \\
\text { aaagtgcacgctactttgctaa }\end{array}$ \\
\hline
\end{tabular}

The letters in lower case stand for the primer sequences used for amplification. Random 71 was generated using the tool: http://usersbirc.au.dk/biopv/php/fabox/random sequence generator.php

In this assay, all the selected ssXNA sequences modulated BACE1 in the same unusual manner i.e. a dose-dependent effect was first observed for the lowest concentrations of oligonucleotides tested followed by an increase in BACE1 activity at the highest concentrations. (Figure $3 \mathrm{~A} 1, \mathrm{~B}, \mathrm{C}$ and $\mathrm{D}$. The loss of inhibition for high concentrations of ssDNA or ssXNA could be due to a loss of accessible oligonucleotides caused by an aggregation phenomenon or due to an activating effect on BACE1 at high concentrations.

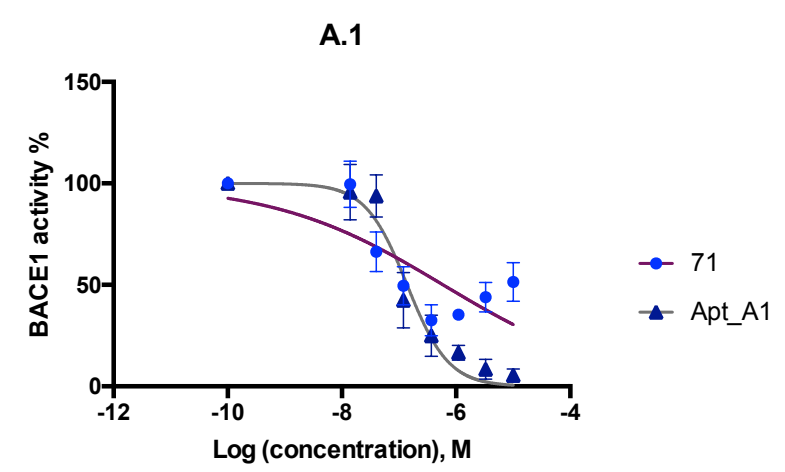


A.2

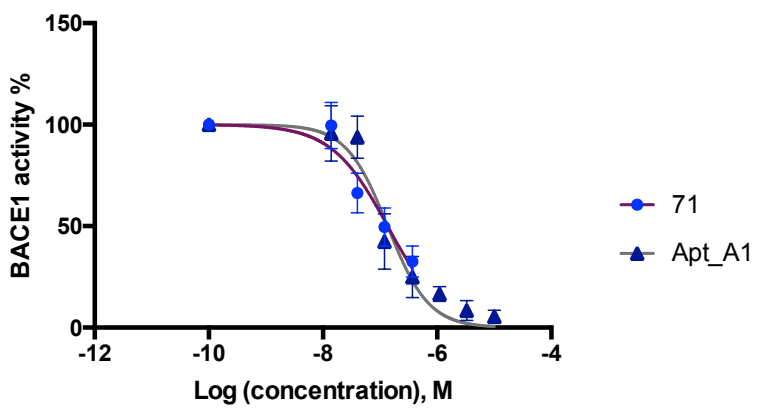

B

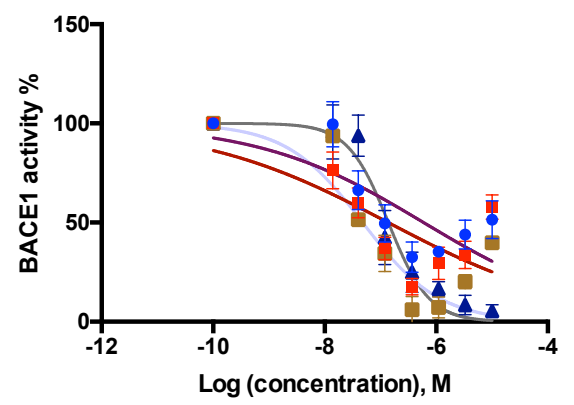

C

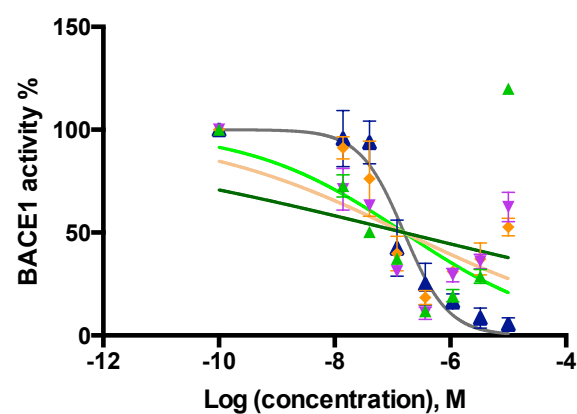

D

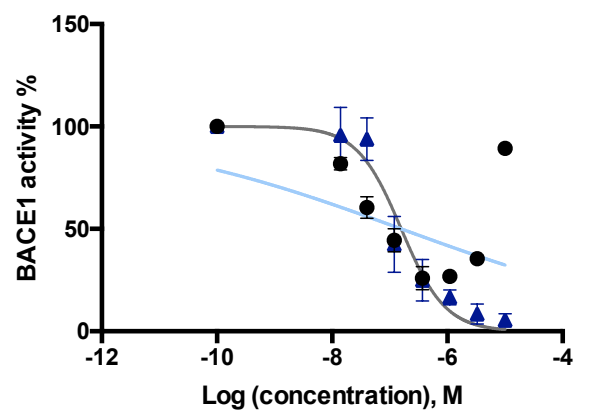

Figure 3. Modulation of BACE1 activity by SSXNA or SSDNA in a FRET assay. For clarity concern, the graphs were split into $A, B, C$ and $D$ panels with Apt A1 as a reference. A2 corresponds to A1 graph without the plot of the highest concentration values for 71 to allow $\mathrm{IC}_{50}$ determination.
- $12 \mathrm{c} 26$

- Apt A1
The results suggested that the XNA sequences had an activating effect at higher concentrations which is not observed with the reference aptamer Apt_A1. However, this trend did not seem to be associated to the presence of chemical modifications nor to the in vitro production method since sequences $71 \mathrm{~T}$ (synthesized by the in vitro production method) and 93 TL (chemically synthesized) also exhibited the same profile. Moreover, at a concentration of $10 \mu \mathrm{M}$, ssXNA 39 produced a slight activating effect instead of a loss of inhibition. At higher concentrations, the selected ssXNA or ssDNA could bind with a lower affinity to an allosteric site. The $\mathrm{IC}_{50}$ values $(95 \% \mathrm{Cl})$ for the tested sequences were estimated without taking into account the data for high concentration of modulator $(1.1 ; 3.3$ and $10 \mu \mathrm{M}$ Figure $3 \mathrm{~A} 2$ ). We found an IC 50 value for Apt_A1 of $138.7 \mathrm{nM}$ that was very close to the value initially described (i.e. 139.81 $\mathrm{nM}$ ) and slightly lower $\mathrm{IC}_{50}$ values (46 to $95 \mathrm{nM}$ ) for all the other sequences (Table 3 ).

Table 3. Activities of representative ssXNA BACE1 inhibitors (and related analogues) isolated by directed evolution.

\begin{tabular}{lll}
\hline aptamer & $\mathrm{IC}_{50}$ & $\mathrm{~K}_{\mathrm{D}}$ \\
\hline 71 & $130.8 \mathrm{nM}(95 \% \mathrm{Cl}: 75.6$ to $226.2 \mathrm{nM})$ & $12.0 \pm 1.2 \mathrm{nM}$
\end{tabular}

71T $\quad 61.9 \mathrm{nM}(95 \% \mathrm{Cl}: 43.7$ to $87.6 \mathrm{nM}) \quad 10.3 \pm 3.7 \mathrm{nM}$

Random $7152.5 \mathrm{nM}$ (95\% Cl: 36.8 to $75.0 \mathrm{nM})$

$3946.5 \mathrm{nM}(95 \% \mathrm{Cl}: 37.2$ to $58.2 \mathrm{nM})$

$93 \quad 52.9 \mathrm{nM}(95 \% \mathrm{Cl}: 39.1$ to $71.5 \mathrm{nM})$

$93 \mathrm{TL} \quad 94.6 \mathrm{nM}(95 \% \mathrm{Cl}: 61.9$ to $144.6 \mathrm{nM})$

$118 \quad 85.4 \mathrm{nM}(95 \% \mathrm{Cl}: 64.1$ to $113.8 \mathrm{nM})$

Apt_A1 $\quad 138.7 \mathrm{nM}(95 \% \mathrm{Cl}: 90.2$ to $213.1 \mathrm{nM})$

From this biochemical analysis, several different hypothetical binding motifs could be inferred for the binding effect of the sequences on BACE1. Indeed, the sequence motif R-RRGRGRCG ( $R=X$ or $T$, motif 1 , Table 2$)$ was common to sequences 71, $71 \mathrm{~T}$ and random 71 (see MEME results in Supplementary data S4.3). On the other hand, sequences 39 and 93 could contain two motifs, one shared with 93TL and another one with 118 that might explain their slightly lower $\mathrm{IC}_{50}$ values. The motif shared between 118, 39 and 93 might expand from the 5'-primer to the core region (motif 3 in green Table 2). This hypothesis could also explain why 93TL exhibited a two times lower activity compared to 93. However, no common motif with Apt_A1 could be clearly identified except a very short CTTGTG motif that could be a start for further truncation investigation (truncated motif 1 in red Table 2). Finally, the variability of the sequences generated from the different SELEX processes and the results of the activity assay suggest that BACE1 displays multiple binding sites for aptameric nucleic acids. 


\section{Conclusions}

To summarize, we have included base-modified triphosphate analogs in the SELEX processes to raise aptamers against betasecretase 1 . The resulting aptamers displayed a remarkable affinity for the target ( $10 \mathrm{nM} K_{d}$ values) with $I_{50}$ values in the low $\mathrm{nM}$ range. In addition, while the selection of ssXNAs was

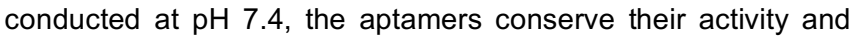
high binding affinities at $\mathrm{pH} 4.5$ which is optimal for the BACE1mediated peptide cleavage activity. ${ }^{[46,47]}$ This feature may be related to allosteric exosite binding rather than a competitive inhibitory effect with the natural substrate; this hypothesis is consolidated by results from BACE1 conformational switch studies based on crystal structure resolution and continuous constant-pH molecular dynamics simulations. ${ }^{[46,38]}$ The ssXNA aptamer identified as sequence 71 not only tightly binds to BACE1 $\left(K_{d}=12 \mathrm{nM}\right)$, but also inhibits its protease activity in vitro in the same range as a previously described unmodified aptamer. ${ }^{[31]}$ Surprisingly, other selected candidates like $n^{\circ} 39$ and 93 were good modulators of BACE1 activity without any detectable binding to BACE1, revealing that strong binding might not be the only crucial parameter determining the capacity of an aptamer to modulate the activity of an enzyme. In contrast, the starting library displayed a significant affinity to BACE1, most likely due to a sequence bias caused by a statistic overrepresentation of strong binders in the starting pool. The newly identified ssXNA aptamer has a similar affinity compared to the same unmodified oligonucleotide despite differences in association and dissociation rate constants. However, all the selected sequences had new features in terms of activity with an unusual behaviour of modulator that was concentrationdependent. Different aptamers with allosteric modulatory activity have already been identified but each aptamer was selective for one conformational state of the target. ${ }^{[48]}$ The vast majority of aptamers described in the literature have an inhibitory effect on their enzymatic target. There are only a few examples of aptamers with an agonist effect on receptors and only one example to the best of our knowledge with an activating effect on thrombin. ${ }^{[49]}$ Even if agonist-antagonist compounds are well known in a drug-receptor interaction context, we are not aware of any aptamer with an inhibitory effect at low concentration and an activating effect at high concentration on its target. Here, it appeared that one ssXNA and the same aptameric sequence could modulate BACE1 activity depending on its concentration. This study paves the road towards more aptamer xenodiversification and the identification of potent inhibitors, especially for the treatment of neurodegenerative diseases. Aptamers have not frequently been used as agents in this field so far, mainly because of the difficult nature of the targets as illustrated by BACE1.

While C5-modified nucleotides have been used for some time in SELEX procedure leading especially to SOMAmers ${ }^{[50]}$ or to alkyne-modified aptamers identification, ${ }^{[51,52]}$ we selected a fully substituted 5-CldU aptamer that can potentially be produced within a bacterium with the use of a specialized polymerase. One unnatural base pair was integrated and replicated for the first time on a plasmid in $E$. coli by Romesberg and colleagues in
$2014 .^{[15]}$ If such a base pair could be stably maintained, it could allow an expansion of the genetic code with numerous applications. ChloroU substitution has been shown to be stably inherited in the chromosome of $E$. coli as an information support. ${ }^{[2]}$ The next step would be to introduce non coding but functional XNA as a selected aptamer in such a living system which function could be controlled exogenously and over time.

\section{Experimental Section}

\section{General}

Synthesis of modified precursors: 5-chloro-2'-deoxyuridine and 7-deaza2 '-deoxyadenosine were converted to their $5^{\prime}$-triphosphates in a one-pot reaction by the Ludwig method. ${ }^{[53,54]}$ DNA sequences: DNA oligonucleotides were purchased from Eurofins (France) and purified by HPLC. The sequences are the following (shown 5' to 3'): FWD-D1 GCCTGTTGTGAGCCTCCTAAC; Bio-REV-D1 (5'BITEG modified) GGGAGACAAGAATAAGCATG; sl-REV-D1 (in bold the 5 '-GC rich reverse repeat sequence with the ability to form stem-loop structure)

CCGCGGGTGGGACTTCCTAGTCCCACCCGCGGGAGACAAGAATAA

GCATG; Bio-FWD-D1 (5'-BITEG modified)

GCCTGTTGTGAGCCTCCTAAC; T3 AATTAACCCTCACTAAAGGG; T7 TATACGACTCACTATAGGG. The single strand oligonucleotide $n^{\circ} 93 \mathrm{TL}$ (5'- GCA CCG GXC GCA GGX XXA GCA AGG XXA CAA GCX GGC AXX AAG GXX GCXC-3') was chemically synthesized by TriLink company.

Human BACE1 ectodomain containing the catalytic domain of the protease (residues 46-460) was kindly provided by Bart de Strooper after production in HEK293T cells (VIB Protein Service Facility, Department of Molecular Biomedical Research Technologiepark 927, B9052 Ghent, Belgium).

Starting materials, reactants and solvents were obtained from SigmaAldrich at a molecular biology grade when available.

Amplification studies with a modified triphosphate precursor: We determined for the D1 DNA library with a random region of 49 bases (5'GCC TGT TGT TGT CCT CCT AAC (N49) CAT GCT TAT TCT TGT CTC CC-3', Metabion, Germany) an extension efficiency of $52 \%$, a complexity of the pool of $8.84 \times 10^{15}$ and an average amplification efficiency of the PCR product of 1.81. D1 library was amplified by using the following optimized conditions: $50 \mathrm{nM}$ template, $3.5 \mathrm{mM} \mathrm{MgCl} 2,200 \mu \mathrm{M}$ dNTP (modified or natural), $1 \mu \mathrm{M}$ forward FWD-D1 and reverse REV-D1-Bio primers, $0.5 \mathrm{mg} / \mathrm{mL}$ BSA, $0.025 \mathrm{U} / \mu \mathrm{L}$ TAQ polymerase (TAQ DNA polymerase Thermus aquaticus $\mathrm{YT}-1$, New England Biolabs), $95^{\circ} \mathrm{C} 1 \mathrm{~min}$, $54^{\circ} \mathrm{C} 1 \mathrm{~min}, 72^{\circ} \mathrm{C} 1.5 \mathrm{~min}, 6$ cycles. These optimal reaction conditions enabled the setting of the large scale PCR that resulted in the generation of 10 copies of the library with a ca. $10^{14}$ complexity. Incorporation of 5CldUTP was also obtained with similar yield by using the AmpiTAQ Gold DNA polymerase (Applied Biosystems) using the same conditions.

In vitro selection: The SELEX methodology outlined below was adapted from the work of Mayer et al. ${ }^{[37]}$ Strand displacement assays: The library used for the selection was obtained after generation of singlestranded XNA. Reverse primer was biotinylated at the $5^{\prime}$ end to enable the removal of the reverse complement by using streptavidin-coated magnetic beads. ${ }^{[35]}$ After immobilization of a $100 \mu \mathrm{L}$ aliquot of the PCRbuilt library on magnetic beads $(0.25 \mathrm{mg}$. Promega) in a $300 \mu \mathrm{L}$ final

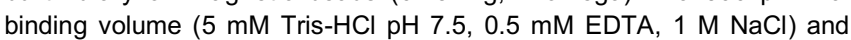
elution of the non-biotinylated strand by addition of $50 \mu \mathrm{L} 0.15 \mathrm{M} \mathrm{NaOH}$ 
followed by $\mathrm{HCl}$ neutralization $(25 \mu \mathrm{L}$ of $0.3 \mathrm{~N} \mathrm{HCl}$ ), approximatively 40 pmol of ssXNA were recovered per aliquot for SELEX A and 15 pmol for SELEX B. Quantitation of the oligonucleotides was done by recording the UV absorption at $260 \mathrm{~nm}$ by using the base composition method for $\varepsilon_{M}$ calculation $\left(\varepsilon_{\mathrm{M}}=9500 \mathrm{M}^{-1} . \mathrm{cm}^{-1}\right.$ for 5 -CldUTP and $12000 \mathrm{M}^{-1} . \mathrm{cm}^{-1}$ for 7 deazadATP) and assuming an identical distribution of the four nucleotides in the library random region._In order to improve the ssXNA production yield and to avoid issues associated to the streptavidin coated beads, ${ }^{[45]}$ an alternative method has been tested by using a 5'phosphorylated reverse primer instead of the biotinylated one for PCR amplification followed by exonuclease $\lambda$ digestion (New England Biolabs, France). ${ }^{[55]}$ Despite several benefits associated to this method including a better yield purification or a less expensive process, we chose to carry on with the streptavidin magnetic beads because of significant drawbacks like remaining dsXNA in the reaction, ${ }^{[56]}$ longer time in duration and reproducibility problems of quantification by UV/Vis spectroscopy. BACE1 immobilization procedure: Firstly, $6.66 \mathrm{nmol}$ BACE1 ectodomain (residues 46-460 containing the catalytic domain of the protease) were incubated on ice with $33.3 \mathrm{nmol}$ EZ-Link Sulfo-NHS-LCBiotin (Thermo Scientific) reagent (in a total volume of $400 \mu \mathrm{L}$ for 30 minutes and further 15 minutes at room temperature. Unbound sulfoNHS-LC-Biotin reagent was removed from the mixture by gel filtration by using P6 spin-columns (BioRad). We obtained a 1:3 ratio mixture of biotinylated and unbiotinylated BACE1 after Western blot determination by using streptavidin beads immobilization experiment (Supplementary data, S2). BACE1 biotinylation mixture $(0.48 \mathrm{nmol}, 50 \mu \mathrm{L})$ was added to $2.5 \mathrm{mg}$ of streptavidin paramagnetic particles (MagneSphere, Promega) in $1 \mathrm{~mL}$ selection buffer $\left(137 \mathrm{mM} \mathrm{NaCl}, 2.7 \mathrm{mM} \mathrm{KCl}, 6.5 \mathrm{mM} \mathrm{Na}_{2} \mathrm{HPO}_{4}\right.$, $1.8 \mathrm{mM} \mathrm{NaH} 2 \mathrm{PO}_{4}, 1.5 \mathrm{mM} \mathrm{MgCl} 2$ and $0.1 \%$ (w/v) BSA, pH 7.4) and incubated for 30 minutes at room temperature under constant agitation. The supernatant was then removed and the beads were washed and resuspended in $1 \mathrm{~mL}$ selection buffer. Uncoated beads were generated by using the same protocol, but without a protein ligand. These beads were used in the counter-selection steps outlined below in order to remove oligonucleotides that may bind non-specifically to the unfunctionalized regions of the beads. In vitro enrichment of ssXNA: The SELEX procedure began with a preincubation of 500 pmol ssXNA for SELEX A and 215 pool for SELEX B with $0.4 \mathrm{mg}$ of non-loaded streptavidin-coated magnetic beads for the counter selection step in a total volume of $400 \mu \mathrm{L}$ selection buffer $(137 \mathrm{mM} \mathrm{NaCl}, 2.7 \mathrm{mM} \mathrm{KCl}, 6.5$ $\mathrm{mM} \mathrm{Na}_{2} \mathrm{HPO}_{4}, 1.8 \mathrm{mM} \mathrm{NaH}_{2} \mathrm{PO}_{4}, 1.47 \mathrm{mM} \mathrm{MgCl}_{2}$ and $0.1 \%(\mathrm{w} / \mathrm{v})$ bovine serum albumin (BSA), pH 7.4) at room temperature. Following incubation the beads were partitioned by using a magnet and the supernatant was immediately added to $0.4 \mathrm{mg}$ BACE1-coated beads in a total volume of $400 \mu \mathrm{L}$ selection buffer for 30 minutes at room temperature. After washing with selection buffer, BACE1-bound sequences were eluted by incubation in $250 \mu \mathrm{L}$ water at $95^{\circ} \mathrm{C}$ for $3 \mathrm{~min}$, concentrated in vacuo and resuspended in $30 \mu \mathrm{L} \mathrm{H}_{2} \mathrm{O}$ and reamplified with suitable primers. The beads were discarded after each round of selection. The first PCR proceeded according to the previously determined conditions in a final volume of $100 \mu \mathrm{L}$. The second PCR proceeded in a $2 \mathrm{~mL}$ final (SELEX A) or $1 \mathrm{ml}$ (SELEX B) volume by the first PCR as the template. After alkaline denaturation of the double-stranded PCR products, filtration on Omega membrane (Nanosep centrifugal devices, Pall Life Sciences), $10 \%$ of desalted "sense" strands were kept aside as safeguard and the rest was introduced to the subsequent rounds of SELEX. Twelve iterations of SELEX were performed. In vitro selection by using nitrocellulose membrane at pH 4.5: SELEX was performed as described by Hall et al. by using nitrocellulose membrane to select aptamers against BACE1 at $\mathrm{pH} 4.5 .^{[37]}$ In every round, prior to selection, ssXNA were thermally equilibrated and refolded in selection buffer by heating to $75^{\circ} \mathrm{C}$ for $3 \mathrm{~min}$ and then slowly cooling to $37^{\circ} \mathrm{C}$ for at least 10 minutes. To minimize nonspecific binding with the nitrocellulose membrane, the ssXNA pool were pre-adsorbed to a $0.45 \mu \mathrm{m}$ nitrocellulose HAWP nitrocellulose disk filter (Analytical Biotechnology, Birkhauser) for $30 \mathrm{~min}$ prior to every round of selection in order to remove filter-binding species. In the first selection cycle, the random ssXNA pool ( 500 pool in $100 \mu \mathrm{L}$ selection buffer at $\mathrm{pH} 4.5$ ) and BACE1 (25 pool in $100 \mu \mathrm{L}$ selection buffer at $\mathrm{pH}$ 4.5) were incubated in a rotating wheel for $30 \mathrm{~min}$ at room temperature. The stringency conditions for the following rounds are described in Supplementary data, S6. The BACE1 bound ssXNA were eluted from the filter with $200 \mu \mathrm{L}$ TE Buffer $\left(7 \mathrm{M}\right.$ urea, $25 \mathrm{mM} \mathrm{EDTA} \mathrm{pH=8)}$ at $95^{\circ} \mathrm{C}$ for $5 \mathrm{~min}$, followed by ethanol precipitation.

Cloning and sequencing analysis: PCR products with unbiotinylated primers FW-D1 and REV-D1 were directly inserted into the TOPO ${ }^{8}$ TA cloning vector (Life Technologies) according to manufacturer instructions. After transforming the loaded vector into competent $\beta 2033$ E. coli K12 strain, ${ }^{[57]}$ the bacteria were plated onto ampicillin selective Petri dishes $\left(10,20,40\right.$ and $200 \mu \mathrm{L}$ transformation) at $37^{\circ} \mathrm{C}$ with an overnight incubation. One hundred and twelve positive clonal colonies for SELEX A, one hundred and eight for SELEX B were randomly picked and grown in liquid LB Amp $(100 \mu \mathrm{g} / \mathrm{ml})$ media and plasmids were purified by using a QIAprep Spin Miniprep Kit according to manufacturer instructions (QIAGEN). An aliquot of all liquid cultures was stored at $-80^{\circ} \mathrm{C}$ in $15 \%$ glycerol final concentration for further production and analysis. The sequencing using the Sanger method was carried on at the sequencing department of Genoscope (Evry, France) by using the T7 and T3 sequencing primers that were incorporated into the TOPO TA vector. The sequences were assessed for quality i.e. proper length and sequence, trimmed to removed primer sequences and then aligned with «Geneious version 6.1.6 (http://www.geneious.com, ${ }^{[40]}$ ) and Muscle alignment tool. ${ }^{[58]}$ For SELEX B, a few sequences were selected to be tested for activity based on MEME results analysis (Supplementary data, S4.1). ${ }^{[59]}$

ssXNA production: dsXNA were first obtained after PCR amplification by a low error rate DNA polymerase (TaKaRa Ex Taq, Ozyme) by applying the following conditions: $2 \mathrm{mM} \mathrm{MgCl} 2$ in the proper $1 \mathrm{x}$ buffer, 0.2 $\mathrm{mM}$ standard dNTP, $0.14 \mathrm{mM}$ modified dNTP, $1 \mu \mathrm{M}$ primers FW-D1 and REV-D1-Bio, $0.025 \mathrm{U} / \mu \mathrm{L}$ TaKaRa Ex Taq, $95^{\circ} \mathrm{C}$ for $30 \mathrm{sec}, 54^{\circ} \mathrm{C}$ for 30 sec, $72^{\circ} \mathrm{C}$ for $1 \mathrm{~min}, 30$ cycles seeded with $4.3 \mathrm{ng} / \mathrm{\mu L}$ of each corresponding purified plasmid as template in a $3.8 \mathrm{~mL}$ total volume. Alkaline denaturation of the PCR product resulted in $\sim 1500$ pmol ssXNA with the use of streptavidin magnetic beads as previously described.

The quality of the ssXNA production process was compared to another method with unequal length strand PCR and PAGE purification. ${ }^{[42]}$ Two $500 \mu \mathrm{L}-\mathrm{PCR}$ were set in parallel. Using the primers FWD-D1 and sI-REV$\mathrm{D} 1$, the unequal length of ssDNA molecules were produced as follows: 2 $\mathrm{mM} \mathrm{MgCl} 2$ in the proper $1 \times$ buffer, $0.2 \mathrm{mM}$ standard dNTP, $1 \mu \mathrm{M}$ primers, $0.025 \mathrm{U} / \mu \mathrm{L}$ TaKaRa Ex Taq seeded with $2150 \mathrm{ng}$ of clone 7.8/60 ( $\left.{ }^{\circ} 71\right)$ purified plasmid from SELEX A as template. PCR products of unequal length strand were analyzed by electrophoresis in a $6 \%$ polyacrylamide 7 $\mathrm{M}$ urea gel and the lower band of interest was purified by electroelution in $0.5 x$ TBE buffer and ethanol precipitation. The same PCR conditions were used for the second $500 \mu \mathrm{L}$ test PCR except the primers FWD-D1 and REV-D1-Bio and the ssDNA were then recovered as previously described after binding and denaturation on magnetic streptavidin coated beads. One method resulted in to 200 pmol of SSDNA and the other method to less than $100 \mathrm{pmol}$, starting from a $500 \mu \mathrm{L}-\mathrm{PCR}$ in both cases. The resulting ssDNA from both conditions were PCR amplified with the corresponding unbiotinylated primers $(50 \mu \mathrm{L}$ final volume, $35 \mathrm{nM}$ as template and 5 cycles), cloned and sequenced (Supplementary data, S7).

The asymmetric PCR procedure was used to produce 5'-biotinylated ssXNA for binding analysis. Using the primers Bio-FWD-D1 and sI-REV$\mathrm{D} 1$, the unequal length of ssDNA molecules were produced as follows: 2 $\mathrm{mM} \mathrm{MgCl} 2$ in the proper $1 \mathrm{x}$ buffer, $0.2 \mathrm{mM}$ standard dNTP, $0.14 \mathrm{mM}$ modified dNTP, $1 \mu \mathrm{M}$ primers, 0.025U/ $\mu \mathrm{L}$ TaKaRa Ex Taq seeded with

For internal use, please do not delete. Submitted_Manuscript 
$4.3 \mathrm{ng} / \mu \mathrm{L}$ of the selected plasmid or $70 \mathrm{nM}$ for the library in a final volume of $1 \mathrm{~mL}$. The mixture was thermally cycled 30 times through $95^{\circ} \mathrm{C}$ for $1 \mathrm{~min}, 37^{\circ} \mathrm{C}$ for $30 \mathrm{~s}$ and $58^{\circ} \mathrm{C}$ for $40 \mathrm{~s}$, and followed by 5 min extension at $58^{\circ} \mathrm{C}$. PCR products of unequal length strand were analyzed by electrophoresis in a $6 \%$ polyacrylamide $7 \mathrm{M}$ urea gel and the lower band of interest was purified by electroelution in $0.5 x$ TBE buffer and ethanol precipitation.

BACE1 MBP-C125APPsw assay: ${ }^{[60]}$ Human BACE1 (1-460): Fc was diluted in reaction buffer $(48 \mathrm{mM}$ ammonium acetate $\mathrm{pH} 4.6,1 \mathrm{mg} / \mathrm{ml}$ BSA and $0.7 \%$ Triton X-100) till a final concentration of $10 \mathrm{nM}$. Substrate fusion protein of maltose binding protein (MBP) and 125 amino acids of the carboxyl terminus of human APP containing Swedish double mutation was diluted till $50 \mathrm{nM}$ in reaction buffer. $30 \mu \mathrm{L}$ test ssXNA was incubated with $50 \mu \mathrm{L}$ substrate and $20 \mu \mathrm{L}$ enzyme at $25^{\circ} \mathrm{C}$ for 3 hours. At the end of the incubation, the reaction mixture was 5-fold diluted in stop buffer (100 mM Tris pH 8.0, $6 \mathrm{mg} / \mathrm{mL}$ BSA and $10 \mathrm{mM}$ Triton X-100). 50 $\mu \mathrm{L}$ of the diluted reaction mixture was then loaded on an ELISA plate precoated with anti-MBP antibody. The cleavage product MBP-C26sw was detected by a neo-epitope antibody against the BACE1 cleavage site. The activity of BACE1 was proportionally related to the amount of cleavage product.

Surface plasmon resonance measurements: Surface plasmon resonance (SPR) measurements were carried out on a Biacore T200 (GE Healthcare, Uppsala, Sweden). In a first set of experiments BACE1 was immobilized on a CM4 chip using standard amine coupling chemistry. To determine the binding kinetics and affinities, biotinylated ssXNA were captured on a commercial streptavidin SA sensor chip (88 RU for the library, $69 \mathrm{RU}$ for 71 and $84 \mathrm{RU}$ for $71 \mathrm{~T}$ ). A reference flow cell was used as a control for non-specific binding and refractive index changes. Buffer blanks were used for double referencing. All interactions were performed in $48 \mathrm{mM}$ ammonium acetate $\mathrm{pH} 4.6,0.7 \%$ Triton $\mathrm{X}-100,137 \mathrm{mM} \mathrm{NaCl}$, $2.7 \mathrm{mM} \mathrm{KCl}$ and $1.5 \mathrm{mM} \mathrm{MgCl}_{2}$. The regeneration conditions applied were $1 \mathrm{M} \mathrm{NaCl}$ and $10 \mathrm{mM} \mathrm{NaOH}$. The estimated affinities and rate constant values resulted from five independent experiments and were determined using the Biacore T200 Evaluation Software 2.0.

Fluorescence resonance energy transfer (FRET) assay: This assay was adapted from $\beta$-Secretase 1 (BACE1) Activity Detection Kit protocol (Sigma-Aldrich, St. Louis, MO). Briefly, all the components were prepared and the assay buffer at $\mathrm{pH} 4.6$ supplemented with $\mathrm{NaCl}, \mathrm{KCl}$ and $\mathrm{MgCl}_{2}$ at final concentrations of $137 \mathrm{mM}, 2.7 \mathrm{mM}$ and $1.47 \mathrm{mM}$, respectively. The BACE1 substrates were diluted down to a concentration of $30 \mu \mathrm{M}$ in this salty assay buffer. BACE1 enzyme was prepared in the same buffer at a concentration of 0.045 units/ $\mu \mathrm{L}$. Stock solutions of test samples were prepared in the appropriate buffer (1650 pmol in $55 \mu \mathrm{L}$ of salty assay buffer) and then serially diluted to give the desired working concentrations ranging from $30 \mu \mathrm{M}$ to $0.014 \mu \mathrm{M}$. $10 \mu \mathrm{L}$ of BACE1 enzyme, substrate, standard, test samples and salty buffer were then added in turn to the wells for a total volume of $30 \mu \mathrm{L}$, with the BACE1 enzyme being added last. 384-wells plates (Corning ${ }^{\mathrm{TM}} 384$ Well Flat Clear Bottom Polystyrene NBS ${ }^{\mathrm{TM}}$ Microplates) were then covered with an adhesive aluminum foil and incubated at $37^{\circ} \mathrm{C}$ for 2 hours. Fluorescence units (FU) were recorded on a fluorometer CLARIOstar (BMG LABTECH GmbH, Ortenberg, Germany) set with excitation at 320 $\mathrm{nm}$ and emission at $405 \mathrm{~nm}$. The percentage of BACE1 activity was calculated by using the following equation: $y=$ FUssXNA/FUo $\times 100$, where FUssXNA and FUo represent the fluorescence units in the presence and absence of ssXNA after subtraction of the blank (substrate in the salty assay buffer). Dose-response curves were created by graphing the percentage of BACE1 activity versus the logarithm of the inhibitor concentration. All experiments were performed in triplicate.

\section{Acknowledgements}

We thank V. Pezo for providing the beta2033 E. coli strain, the sequencing department of the Genoscope, Evry and M. Hollenstein for improving the manuscript. This work was supported by the European Science Foundation under the EuroSYNBIO program (SYNAPTA project), the European Research Council under the European Union's Seventh Framework Program (FP7/2007-2013)/ERC Grant agreement no. ERC-2012-ADG_20120216/320683 and by the University d'Evry-Val-d'Essonne through the 'Fonds de Rayonnement pour la Recherche' (Aptalzheimer project).

Keywords: XNA • modified aptamer - BACE1 secretase inhibitor

[1] S. M. Morris, Mutat. Res. Genet. Toxicol. 1993, 297, 39-51.

[2] P. Marlière, J. Patrouix, V. Döring, P. Herdewijn, S. Tricot, S. Cruveiller, M. Bouzon, R. Mutzel, Angew. Chemie - Int. Ed. 2011, 50, 7109-7114.

[3] D. B. Dunn, J. D. Smith, Biochem. J. 1957, 67, 494-506.

[4] S. Zamenhof, B. Reiner, K. De Giovanni, R., \& Rich, J. Biol. Chem. 1956, 219, 165-173.

[5] F. Jaunin, A. E. Visser, D. Cmarko, J. A. Aten, S. Fakan, J. Histochem. Cytochem. 1998, 46, 1203-1209.

[6] T. Yokochi, D. M. Gilbert, Curr. Protoc. Cell Biol. 2007, Chapter 22, Unit 22.10.

[7] C. H. Kim, A. Darwanto, J. A. Theruvathu, J. L. Herring, L. C. Sowers, Chem. Res. Toxicol. 2010, 23, 740-748.

[8] E. Eremeeva, M. Abramov, L. Margamuljana, J. Rozenski, V. Pezo, P. Marlière, P. Herdewijn, Angew. Chemie - Int. Ed. 2016, 55, 7515-7519.

[9] G. Acs, E. Reich, Antibiotics 1967, 494-498.

[10] A. Bloch, R. J. Leonard, C. A. Nichol, Biochim. Biophys. Acta 1967, 138, 10-25.

[11] E. Eremeeva, M. Abramov, P. Marlière, P. Herdewijn, Org. Biomol. Chem. 2017, 15, 168-176.

[12] A. Patra, J. Harp, P. S. Pallan, L. Zhao, M. Abramov, P. Herdewijn, M. Egli, Nucleic Acids Res. 2013, 41, 2689-2697.

[13] A. H. El-Sagheer, A. P. Sanzone, R. Gao, A. Tavassoli, T. Brown, Proc. Natl. Acad. Sci. 2011, 108, 11338-11343.

[14] A. P. Sanzone, A. H. El-Sagheer, T. Brown, A. Tavassoli, Nucleic Acids Res. 2012, 40, 10567-10575.

[15] D. A. Malyshev, K. Dhami, T. Lavergne, T. Chen, N. Dai, J. M. Foster, I. R. Corrêa, F. E. Romesberg, Nature 2014, 509, 385-388.

[16] J. Zhou, J. Rossi, Nat. Rev. Drug Discov. 2016, 16, 181-202.

[17] J. C. Rohloff, A. D. Gelinas, T. C. Jarvis, U. A. Ochsner, D. J. Schneider, L. Gold, N. Janjic, Mol. Ther. - Nucleic Acids 2014, 3, e201.

[18] B. N. Gawande, J. C. Rohloff, J. D. Carter, I. von Carlowitz, C. Zhang, D. J. Schneider, N. Janjic, Proc. Natl. Acad. Sci. 2017, 114, 2898-2903.

[19] K. Sefah, Z. Yang, K. M. Bradley, S. Hoshika, E. Jimenez, L. Zhang, G. Zhu, S. Shanker, F. Yu, D. Turek, et al., Proc. Natl. Acad. Sci. 2014 $111,1449-1454$.

[20] M. Kimoto, R. Yamashige, K. I. Matsunaga, S. Yokoyama, I. Hirao, Nat. Biotechnol. 2013, 31, 453-457.

[21] N. K. Vaish, R. Larralde, A. W. Fraley, J. W. Szostak, L. W. McLaughlin, Biochemistry 2003, 42, 8842-8851.

[22] S. Diafa, M. Hollenstein, Molecules 2015, 20, 16643-16671.

[23] M. Renders, E. Miller, C. H. Lam, D. M. Perrin, Org. Biomol. Chem. 2017, 15, 1980-1989.

[24] F. Lipi, S. Chen, M. Chakravarthy, S. Rakesh, R. N. Veedu, RNA Biol. 2016, 13, 1232-1245.

[25] S. W. Santoro, G. F. Joyce, K. Sakthivel, S. Gramatikova, C. F. Barbas, J. Am. Chem. Soc. 2000, 122, 2433-2439. 
[26] A. V. Sidorov, J. A. Grasby, D. M. Williams, Nucleic Acids Res. 2004, 32, 1591-1601.

[27] M. Hollenstein, C. J. Hipolito, C. H. Lam, D. M. Perrin, ChemBioChem 2009, 10, 1988-1992.

[28] A. I. Taylor, V. B. Pinheiro, M. J. Smola, A. S. Morgunov, S. Peak-Chew, C. Cozens, K. M. Weeks, P. Herdewijn, P. Holliger, Nature 2015, 518, 427-430.

[29] S. Barão, D. Moechars, S. F. Lichtenthaler, B. De Strooper, Trends Neurosci. 2016, 39, 158-169.

[30] A. Rentmeister, A. Bill, T. Wahle, J. Walter, M. Famulok, Rna 2006, 12, 1650-1660.

[31] H. Liang, Y. Shi, Z. Kou, Y. Peng, W. Chen, X. Li, S. Li, Y. Wang, F. Wang, X. Zhang, PLoS One 2015, 10, 1-14

[32] M. Cully, Nat. Rev. Drug Discov. 2014, 13, 804-804.

[33] R. Vassar, P. H. Kuhn, C. Haass, M. E. Kennedy, L. Rajendran, P. C Wong, S. F. Lichtenthaler, J. Neurochem. 2014, 130, 4-28.

[34] J. Müller, B. Isermann, C. Dücker, M. Salehi, M. Meyer, M. Friedrich, T. Madhusudhan, J. Oldenburg, G. Mayer, B. Pötzsch, Chem. Biol. 2009 16, 442-451.

[35] M. S. L. Raddatz, A. Dolf, E. Endl, P. Knolle, M. Famulok, G. Mayer Angew. Chemie - Int. Ed. 2008, 47, 5190-5193.

[36] G. Mayer, M.-S. L. Ahmed, A. Dolf, E. Endl, P. A. Knolle, M. Famulok, Nat. Protoc. 2010, 5, 1993-2004.

[37] G. Mayer, T. Höver, Nucleic Acid Pept. Aptamers Methods Protoc. 2009, 19-32.

[38] C. R. Ellis, J. Shen, J. Am. Chem. Soc. 2015, 137, 9543-9546.

[39] B. Hall, S. Arshad, K. Seo, C. Bowman, S. D. Jhaveri, A. D. Ellington, 2009, 1-27.

[40] M. Kearse, R. Moir, A. Wilson, S. Stones-Havas, M. Cheung, S. Sturrock, S. Buxton, A. Cooper, S. Markowitz, C. Duran, Bioinformatics 2012, 28, 1647-1649.

[41] Timothy L. Bailey and Charles Elkan, Proc. Second Int. Conf. Intell. Syst. Mol. Biol. AAAI Press. Menlo Park. California, 1994, 28-36.

[42] X. Cao, S. Li, L. Chen, H. Ding, H. Xu, Y. Huang, J. Li, N. Liu, W. Cao, Y. Zhu, Nucleic Acids Res. 2009, 37, 4621-4628.
[43] Y. Wang, N. Ng, E. Liu, C. H. Lam, D. M. Perrin, Org. Biomol. Chem. 2017, 15, 610-618.

[44] S. Sinha, J. P. Anderson, Nature 1999, 402, 537.

[45] A. Paul, M. Avci-Adali, G. Ziemer, H. P. Wendel, Oligonucleotides 2009 19, 243-254.

[46] H. Shimizu, A. Tosaki, K. Kaneko, T. Hisano, T. Sakurai, N. Nukina, Mol. Cell. Biol. 2008, 28, 3663-3671.

[47] F. Grüninger-Leitch, D. Schlatter, E. Küng, P. Nelböck, H. Döbeli, J. Biol. Chem. 2002, 277, 4687-4693.

[48] A. W. Kahsai, J. W. Wisler, J. Lee, S. Ahn, C. I. TJ, S. M. Dennison, D. P. Staus, A. R. Thomsen, K. M. Anasti, B. Pani, Nat. Chem. Biol. 2016, 12, 709-716.

[49] X. Tan, S. K. Dey, C. Telmer, X. Zhang, B. A. Armitage, M. P. Bruchez, ChemBiochem 2014, 15, 205-208.

[50] L. Gold, D. Ayers, J. Bertino, C. Bock, A. Bock, E. N. Brody, J. Carter, A B. Dalby, B. E. Eaton, T. Fitzwater, et al., PLoS One 2010, 5, e15004.

[51] I. S. MacPherson, J. S. Temme, S. Habeshian, K. Felczak, K. Pankiewicz, L. Hedstrom, I. J. Krauss, Angew. Chemie - Int. Ed. 2011 50, 11238-11242.

[52] J. S. Temme, M. G. Drzyzga, I. S. MacPherson, I. J. Krauss, Chem. - A Eur. J. 2013, 19, 17291-17295.

[53] J. Ludwig, Acta Biochim. Biophys. Acad. Sci. Hung 1981, 16, 131-133.

[54] J. D. Hoheisel, H. Lehrach, FEBS Lett. 1990, 274, 103-106.

[55] M. Citartan, T. H. Tang, S. C. Tan, S. C. B. Gopinath, World J. Microbiol. Biotechnol. 2011, 27, 1167-1173.

[56] M. Svobodová, a Pinto, P. Nadal, C. K. O' Sullivan, Anal. Bioanal. Chem. 2012, 404, 835-42.

[57] C. Parsot, EMBO J. 1986, 5, 3013-9.

[58] R. C. Edgar, Nucleic Acids Res. 2004, 32, 1792-1797.

[59] T. L. Bailey, M. Boden, F. A. Buske, M. Frith, C. E. Grant, L. Clementi, J. Ren, W. W. Li, W. S. Noble, Nucleic Acids Res. 2009, 37, W202-W208.

[60] L. Zhou, L. Chávez-Gutiérrez, K. Bockstael, R. Sannerud, W. Annaert, P. C. May, E. Karran, B. De Strooper, J. Biol. Chem. 2011, 286, $8677-$ 8687. 